\title{
Study on Traveler Oriented Dynamic Traffic Assignment Problems
}

\author{
Huifang Deng ${ }^{1}$, and Hao Cheng ${ }^{1}$
}

\begin{abstract}
In the previously published work [1], we proposed a traveler oriented model which incorporates the traveler dynamics into the cell transmission model (TOM-CTM). By taking travelers as the source of the traffic load, this model can be used to enhance existing CTMs. An open source macro-simulation tool (AuroraRNM), is used for experiments on a real road network. The results show that the TOM-CTM can reflect traffic dynamics properly and reasonably, provides a way to find out the manageable status in a nonequilibrium traffic, and is more practical than user or system optimal solutions. Based on this model, in this extended version, we explored the effect of historical traffic information (HTI) on the traffic. We discussed an HTI guided model (HTIGM) which uses the time dependent shortest path as the guidance. The further simulation results show that the HTIGM can provide another manageable way to guide the traffic flow by influencing the travelers' choices and can be also used to spot the bottlenecks in road networks.
\end{abstract}

Keywords-Dynamic Traffic Assignment, Cell transmission model, Traveler dynamics, Nonequilibrium traffic, Historical traffic information.

\section{INTRODUCTION}

HE dynamic traffic assignment (DTA) [2] model is one $\mathrm{T}$ of the most important parts within a modern intelligent transportation system (ITS). Before the DTA model was proposed, the static traffic assignment model was widely adopted. But with the ever growing travel demand, the traditional static traffic model has failed to catch the traffic dynamics. Meanwhile, the imbalance between the demand and limited supply has become more and more serious, especially during the rush hours. Therefore the DTA model subsequently emerged, which focuses on studying the dynamic behaviors of the traffic flow.

Since Merchant and Nemhauser [3] first proposed a mathematical programming DTA model in 1978, the DTA problem has been studied for three decades. The model described in their seminal work, generally called the M-N model, treats the DTA problem as a system optimum problem with fixed demand, single commodity and single destination. After that, many works have been done to further develop the DTA theory. Peeta and Ziliaskopoulos [4] published a review article about DTA which described pre-2001's works in details. It can be seen through the article that the models proposed during that period are almost homogeneous models, which implies all vehicles are sharing the same characteristics,

\footnotetext{
${ }^{1}$ School of Computer Science and Engineering, South China University of Technology, Guangzhou, China.
}

such as speed, size, capacity and so on. But the real world traffic is much more complicated, one of the most obvious scenarios that can be directly observed is multiple type of vehicles running on the road with different speeds. Due to the development of technologies [5], some researchers have noticed the emerging new challenges. Zheng and Arulselvan [6] modeled managed lanes as a system optimum mixed integer nonlinear programming problem based on the work of the literature [7]. Gangi [8] proposed a multiclass model incorporating different type of vehicles for estimating an evacuation plan. For general road network, Tuerprasert and Aswakul [9] enhanced the original cell transmission model (CTM) $[10,11]$ to support multiple vehicle classes. There are also some simulation tools developed by universities, like DynaMIT [12] and AuroraRNM [13,14], which have been incorporated basic multiclass dynamics into models. Besides considering multiple vehicles, some researchers studied other aspects of transportation, such as the route or mode choices $[15,16]$, the uncertain traffic demand [17], the effects of different departure time [18], and the simultaneous departure time/route choices [19, 20]. Reference [21] reviewed the model classification and travel choices adopted in DTA in recent years.

In this extended version, we explored the effect of historical traffic information on the traffic based on the model we proposed in the early published paper [1]. Different from the previous works, we first formulate the relationship between travelers and traffic. Then, we believe that with the guidance of historical traffic information (HTI) the traffic can be assigned more reasonable, therefore, we discuss how to incorporate HTI into our model and how it influences the traffic. All these considerations are implemented from the travelers' perspective. This paper reveals that the management strategy taken by the traffic management center (TMC) will first influence or determine travelers' choices, then the composition of vehicles on roads, and finally the traffic flow. This is quite different from the previous approaches that normally directly dealt with the traffic flow and ignored the relationship between travelers and traffic. Enclosure of such factors in the study with our model would enable TMCs to reassign and analyze traffic more easily and controllably, and help them to find out which method is most suitable to manage traffic. The experimental results backs up the above statements as demonstrated in Section III.

The structure of this paper is as follows. The Section I is the introduction. The Section II gives descriptions and formulations of the model in details. The Section III deals with the numerical simulation and results analysis. The conclusions 
come with the last Section.

\section{MODEL DESCRIPTIONS}

\section{A. Traveler Oriented Multiclass Cell Transmission Model (TOM-CTM)}

In this section, the model proposed in [1] is presented before we could further discuss the effect of historical traffic information. Different from conventional CTM, demand in the TOM-CTM is divided into two parts: the traffic demand and the travel demand. The relationship between these two demand during each planning interval is formulated as equation (1), where $\hat{d}_{r s}^{t}(u)$ denotes the total demand of travelers from cell $r$ to cell $s$ that depart at $t$ taking vehicle $u$ and $d_{r s}^{t}(u)$ as the total demand of vehicles. Equation (1) means if travelers decide to travel with non-periodical vehicles (represented by ${ }_{u}=0$ ), they may start their trips immediately and the traffic demand is equal to the number of travelers divided by the capacity ${ }_{u}$ of each vehicle. Conversely, a periodical vehicle class, like a bus, has its own departing schedule that is static for a quite long time in spite of travelers' quantities.

$$
d_{r s}^{t}(u)=\left\{\begin{array}{c}
\left\lceil\frac{\hat{d}_{r s}^{t}(u)}{u},_{u}=0\right. \\
\left\lfloor\frac{t \times}{u}\left\lfloor\frac{(t 1) \times}{u}\right\rfloor\right\rfloor,{ }_{u}>0
\end{array}\right.
$$

In order to simulate travelers' boarding and dropping behaviors, additional features are added to conventional CTM. Fig. 1 shows a typical cascading scenario in a road network and travelers are assumed evenly distributed on the road and in the vehicles. More complex scenarios like merging and diverging can be easily formulated from it. In the figure, the solid lines show the traffic direction and $\hat{y}_{i j}^{u}(t)$ above the lines denotes the number of travelers traveling from cell to cell. Based on the evenly distributed assumption, $\hat{y}_{i j}^{u}(t)$ is calculated by equation (2), where $N_{i}^{u}(t)$ denotes the number of travelers in $n_{i}^{u}(t)$ vehicles and $y_{i j}^{u}(t)$ denotes the traffic flow.

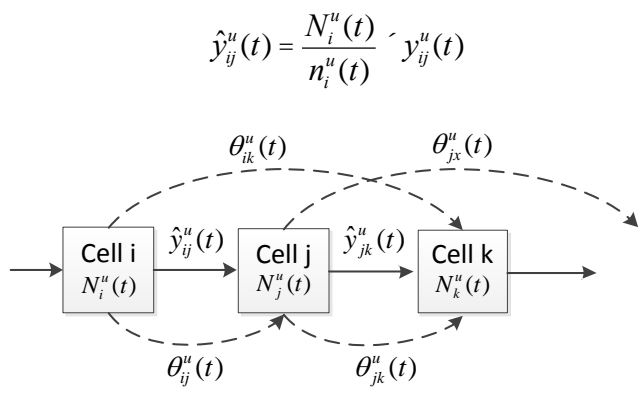

Fig. 1: The flow of travelers and vehicles between cells.

$n_{i}^{u}(t)$ and $y_{i j}^{u}(t)$ in equation (2) can be calculated based on any CTM implemented with multiclass characteristics, like [9] or [14]. For $N_{i}^{u}(t)$, it is updated by equation (3) according to three parts: the travelers leaving the cell, the valid travelers entering the cell and the new travel demand.

$$
\begin{aligned}
& N_{j}^{u}(t+1)=N_{j}^{u}(t) \quad \hat{y}_{j k}^{u}(t)+\left[\begin{array}{ll}
1 & { }_{i j}^{u}(t)
\end{array}\right] \quad \hat{y}_{i j}^{u}(t)+ \\
& \min \left\{\quad \begin{array}{lllll}
x_{u}(i) & & i+1 \\
i x & (u) \hat{d}_{i x}^{t+1}, \quad & & n_{i}^{u}(t+1) & \left.N_{j}^{u}(t+1)\right\}
\end{array}\right.
\end{aligned}
$$

Similar to $N_{i}^{u}(t), \quad{ }_{i j}^{u}(t)$ is also updated by equation (4) according to three parts. The key point is to recalculate the number of travelers heading to each following station according to the in-travelers, out-travelers and new-travelers.

$$
{ }_{j x}^{u}(t+1)=\frac{{ }_{j x}^{u}(t) \times\left[N_{j}^{u}(t)\right.}{\left.\hat{y}_{j k}^{u}(t)\right]+{ }_{i x}^{u}(t) \times \hat{y}_{i j}^{u}(t)+{ }^{\prime}}
$$

Where the new travelers increment ' is calculated by equation (5) in which ${ }_{r s}^{t}(u)$ denotes the proportion of travellers from cell $r$ to $s$ at time $t$ choosing vehicle class $\mathrm{u}$.

$$
\begin{aligned}
\prime= & \frac{{ }_{i x}^{t+1}(u) \hat{d}_{i x}^{t+1}}{\sum_{y \in \Re_{u}(i)}{ }_{i y}^{t+1}(u) \hat{d}_{i y}^{t+1}} \times \\
& \min \left\{\sum_{x \in \Re_{u}(i)}{ }_{i x}^{t+1}(u) \hat{d}_{i x}^{t+1}, \quad{ }_{u} \times n_{i}^{u}(t+1) \quad N_{j}^{u}(t+1)\right\}
\end{aligned}
$$

More information about TOM-CTM can be found in reference [1].

\section{B. Historical Traffic Information}

In order to study how historical traffic information exerting an effect on traffic, let us consider the following scenario. ITS is used to help us find out which routes are congested constantly through analyzing the historical traffic data, and then provides the information to the travelers as a guidance. A proportion of travelers are very likely to choose different routes according to the guide in order to bypass those congested roads. It is based on the belief that if a specific route is congested for some period of time, it is more likely to be congested again for the same period of time in the following days because most of travel demand is derived from travelers' daily regular activities, such as going to work from home or vice versa. Let us call this regularity as the traffic pattern. Obviously, the travelers' choices determine the traffic pattern, and the traffic pattern will, in return, affect the travelers' choices.

One intuitive way to guide travelers is to provide historical shortest paths with different departure time that is called the time dependent shortest path (TDSP). But to quickly obtain the TDSP is not an easy job and needs a powerful capability of analyzing the huge historical traffic data collected by various sensor systems, so we implement a distributed algorithm to solve the all-to-one TDSP problem for all departure time based on the dynamic shortest path optimality conditions [22]. The algorithm is implemented by MapReduce[23] which makes the algorithm easy to deploy on a convenient and scalable data processing platform. The algorithm details will not be discussed in this paper due to limited pages, but how it works in a historical information guided model (HTIGM) will be included in the experimental section. Fig. 2 shows the complete procedure of HTIGM. 
1. InverseAdjacencyListMR is a MapReduce procedure that converts the data structure in the file containing the cost of each link to the adjacency list.

2. The adjacency list is taken as the input of TDSP algorithm in our works, and the time dependent results are passed to the next module. Only directly adjacent node on the shortest path is stored here rather than full path's.

3. ShortestPathResultsConverter extracts and caches the full shortest path between each two nodes from above results.

4. ShortestPathAdapter gets the specific shortest path from cache according to the query.

5. TntpFileProcessor calculates split ratios according to the origin-destination (OD) demand and travelers' choices (such as $20 \%$ of travelers accepting the historical information), and output an AuroraRNM configuration file in term of network topology, OD demand and split ratios.

6. AuroraRNM loads the configuration file and runs the simulation. Its results are stored as the input for the first step, and then do it all over again according to the travelers' choices.

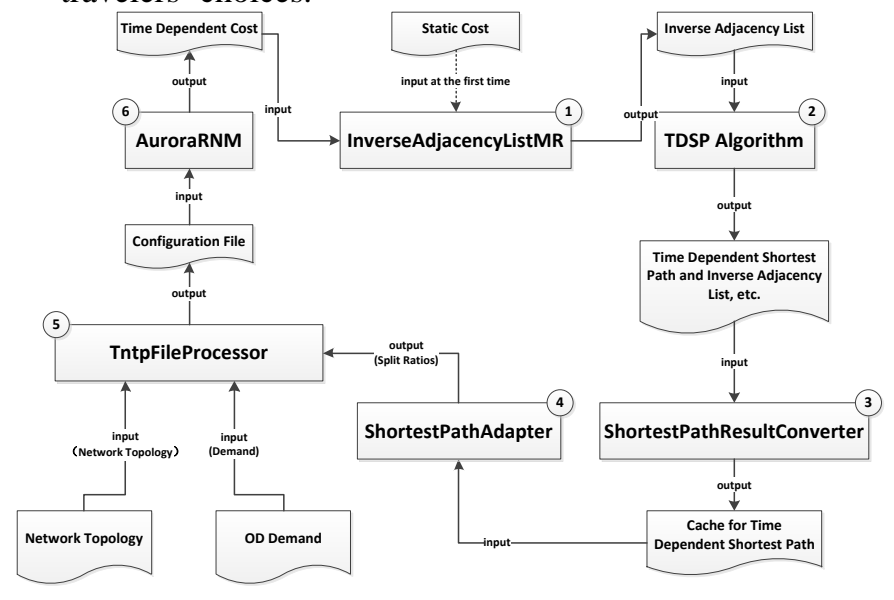

Fig. 2: Implementation procedure of HTIGM.

\section{MODEL STUDY}

For simplicity, it is presumed that the travel time is the most concerns of all travelers. It means that travelers will preferably choose vehicles that run on the corresponding shortest path. Then the simulation results of above traffic pattern will be regarded as the historical traffic information for the subsequent studies. The Sious-Falls network [24] is used for experiments, which is a typical test network adopted in many literatures. This network consists of 24 nodes and 76 links. On-ramps and off-ramps are added in order to handle the demand entering and leaving the network. In the following experiments, we discuss on how the proportion of travelers who accept the historical information changing influences the traffic status.

Fig. 3 shows the delays for different proportions, where HTIGM_20 denotes there are $20 \%$ of travelers following the historical information guide, i.e., these travelers following the time dependent shortest paths, but the $80 \%$ of travelers sticking to their choices. Because HTIGM_0 means no one accepts the information, its results are exactly the same as before. It can be seen from the figure when there are $40 \%$ of travelers choosing to follow the shortest path calculated from the historical traffic data, the delay of the entire road network is effectively reduced. After that, even more travelers choosing to follow the guide will not have obvious effects.

The heavy delay in HTIGM_0 is not only because there are too many vehicles on some routes, but also some of congested routes can be run through rapidly but cannot bear too much traffic load. Therefore, more travelers following the guide will help the traffic flow avoid these bottlenecks and finally reduce the delay. But delay only reflect how congested a road is, when more than $40 \%$ of travelers following the guide, the total travel time will definitely fluctuate because more and more travelers take circuitous routes.

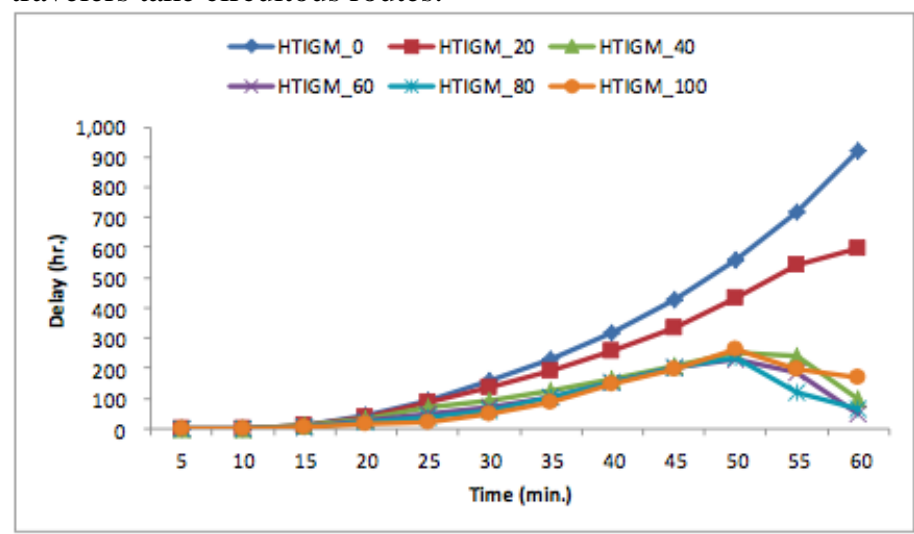

Fig. 3: Comparisons of different choices' delays

Results in Fig. 4 verified above conjecture, with the increasing proportion of travelers following the guide, vehicle hours traveled decrease at first before $40 \%$ because the congestions are alleviated and the total extra time increased due to bypassing is lower than the waiting time. But if the increase continues, the roads congested before become clear and more travelers choose the relatively long way, which finally result in the increase of the total travel time.

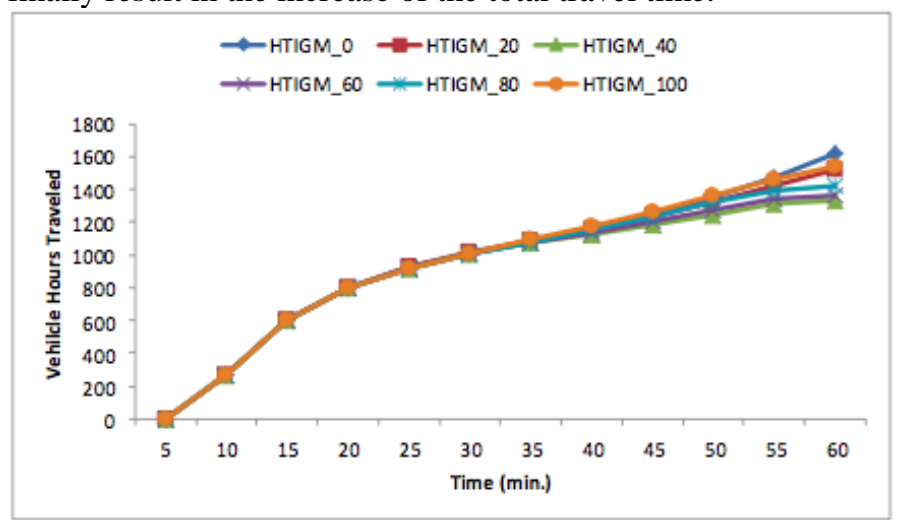

Fig. 4: Vehicle hours traveled of different choices 


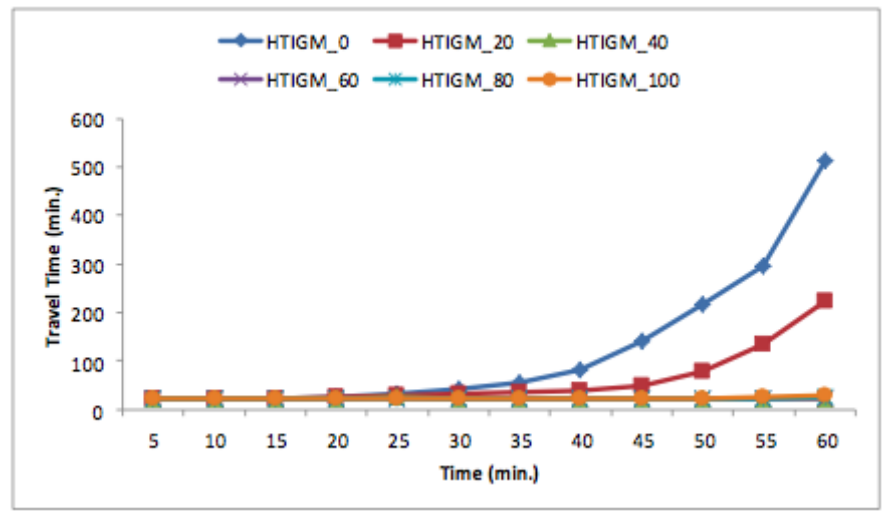

Fig. 5: Travel time on the static shortest path of node $19 \rightarrow 1$ under free flow

Fig. 5 and Fig. 6 show varieties of travel time of two shortest paths from node 19 to node 1 respectively, where the former belongs to the original static shortest path under free flow, and the latter belongs to the new time dependent shortest path related to the historical information. It can be seen from these two figures, that is because more and more travelers follow the new shortest path, the traffic status of the original shortest path is alleviated and keep steady after $40 \%$, whereas the new shortest path gathers more traffic flow, and finally becomes congested around 50 minutes. However the new path can bear more traffic load than the original, so it gets congested only when more than $80 \%$ of travelers choose it. This shows us the bottleneck in current road network and explains the reason on why delays have no big change after $40 \%$.

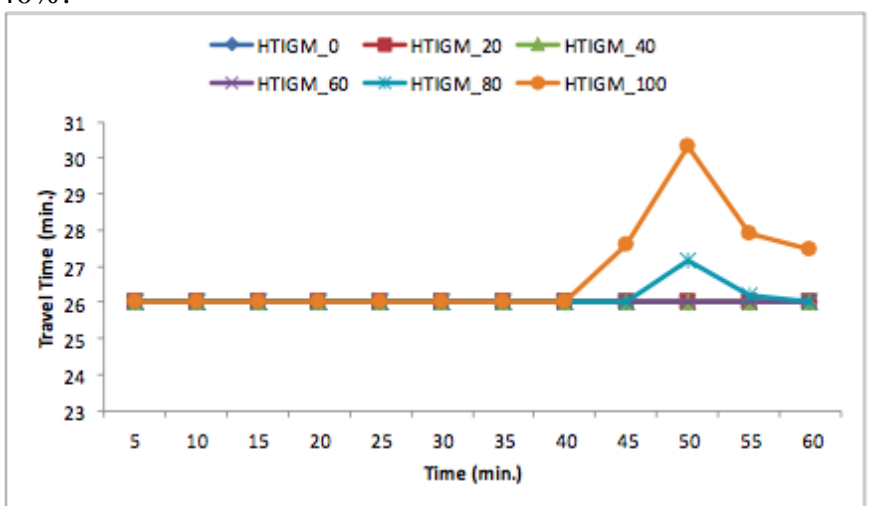

Fig. 6: Travel time on the time dependent shortest path of note $19 \rightarrow 1$

\section{CONCLUSION}

The real world's traffic is hard to reach the equilibrium status, like user optimal or system optimal status. Because in order to operate the optimal solution to the real traffic, many actions along with manpower, physical and financial resources should be taken. The proposed model is more practical for the TMC to apply not only because it can reach an acceptable status based on the nonequilibrium traffic, but also because it catches dynamics of the key subject of the traffic management (i.e., travelers). In the real world, the traffic managers could leverage the statistics of travelers, such as total demand, vehicle preferences, public transit schedules, etc., then use the model to predicate traffic load and find out the most appropriate status (when no more efforts are necessary to put in order to get some minor improvements) they can make. For example, 2 travelers per vehicle would be the most appropriate choice when planning the HOV lanes in the first scenario, and guide $40 \%$ of travelers to other routes according to the historical information to minimize the congestion in another scenario.

However, the decoupled demand in the TOM-CTM needs more calculation work for preparing traffic data, which is almost an unrealizable job before. But the latest advances in IT technologies bring forward the possibilities. Traffic data now can be easily collected, stored and processed with the help of the technologies such as the Internet of Things and the Cloud Computing. The HTIGM does show the feasibility to apply the historical traffic information to the real world. However, only the historical information is not enough to generate the automatic guide which does not need the intervention of the traffic management center, as travelers themselves are unable to know the proportions of choices at any time without real time information. This issue will be addressed in future works.

\section{REFERENCES}

[1] Huifang Deng and Hao Cheng, "Traveler Oriented Dynamic Traffic Assignment Based on Multiclass Cell Transmission Model," 2013 International Conference on Connected Vehicles and Expo (ICCVE), pp. 60-65, 2013. http://dx.doi.org/10.1109/ICCVE.2013.6799770

[2] Y.-C. Chiu et al., "Dynamic Traffic Assignment: A Primer," Transportation Research E-Circular, vol. E-C153, June 2011.

[3] D. K. Merchant and G. L. Nemhauser, "A Model and an algorithm for the dynamic traffic assignment problems," Transportation Science, vol. 12, no. 3, pp. 183-199, 1978. http://dx.doi.org/10.1287/trsc.12.3.183

[4] S. Peeta and A.K. Ziliaskopoulos, "Foundations of Dynamic Traffic Assignment: The Past, the Present and the Future," Networks and Spatial Economics, vol. 1, no. 3, pp. 233-265, 2001. http://dx.doi.org/10.1023/A:1012827724856

[5] Bin Ran, Peter J. Jin, David Boyce, Tony Z. Qiu, and Yang Cheng, "Perspectives on Future Transportation Research: Impact of Intelligent Transportation System Technologies on Next-Generation Transportation Modeling," Journal of Intelligent Transportation Systems, vol. 16, no. 4, pp. 226-242, 2012. http://dx.doi.org/10.1080/15472450.2012.710158

[6] Qipeng P. Zheng and Ashwin Arulselvan, "Discrete time dynamic traffic assignment models and solution algorithm for managed lanes," J. Glob Optim., vol. 51, no. 1, pp. 47-68, 2011. http://dx.doi.org/10.1007/s10898-010-9618-5

[7] Artyom Nahapetyan and Siriphong Lawphongpanich, "Discrete-time dynamic traffic assignment models with periodic planning horizon: system optimum," J. Glob Optim., vol. 38, no. 1, pp. 41-60, 2007. http://dx.doi.org/10.1007/s10898-006-9082-4

[8] M. Di Gangi, "Modeling Evacuation of a Transport System: Application of a Multimodal Mesoscopic Dynamic Traffic Assignment Model," IEEE Transactions on Intelligent Transportation Systems, vol.12, no.4, pp.1157-1166, Dec. 2011. http://dx.doi.org/10.1109/TITS.2011.2143408

[9] Kamonthep Tuerprasert and Chaodit Aswakul, "Multiclass Cell Transmission Model for Heterogeneous Mobility in General Topology of Road Network," Journal of Intelligent Transportation Systems, vol. 14, no. 2, pp. 68-82, 2010. http://dx.doi.org/10.1080/15472451003719715

[10] C. F. Daganzo, "The cell transmission model: A dynamic representation of highway traffic consistent with the hydrodynamic theory," Transportation Research Part B, vol. 28, no. 4, pp. 269-287, 1994. http://dx.doi.org/10.1016/0191-2615(94)90002-7

[11] C. F. Daganzo, "The cell transmission model, Part II: Network traffic," Transportation Research Part B, vol. 29, no. 2, pp. 79-93, 1995. http://dx.doi.org/10.1016/0191-2615(94)00022-R 
[12] M. Milkovits, E. Huang, C. Antoniou, M. Ben-Akiva, and J.A. Lopes, "DynaMIT 2.0: The Next Generation Real-Time Dynamic Traffic Assignment System," Advances in System Simulation, 2010 Second International Conference on, pp.45-51, Aug. 2010. http://dx.doi.org/10.1109/simul.2010.28

[13] Aurora RNM [Online]. Available: http://code.google.com/p/aurorarnm.

[14] A.A. Kurzhanskiy and P. Varaiya, "Using Aurora Road Network Modeler for Active Traffic Management," American Control Conference, pp. 2260-2265, 2010. http://dx.doi.org/10.1109/acc.2010.5530532

[15] Zhen (Sean) Qian and H. Michael Zhang, "A Hybrid Route Choice Model for Dynamic Traffic Assignment," Networks and Spatial Economics, vol. 13, no. 2, pp. 183-203, 2012.

[16] Sho-ichiro Nakayama, Jun-ichi Takayama, Junya Nakai, and Kazuki Nagao, "Semi-dynamic traffic assignment model with mode and route choices under stochastic travel times," Journal of Advanced Transportation, vol. 46, no. 3, pp. 269-281, 2012. http://dx.doi.org/10.1002/atr.208

[17] B. D. Chung, T. Yao and B. Zhang, "Dynamic Traffic Assignment under Uncertainty: A Distributional Robust Chance-Constrained Approach," Networks and Spatial Economics, vol. 12, no. 1, pp. 167181, 2012. http://dx.doi.org/10.1007/s11067-011-9157-8

[18] H. Kim, Y. Lim and S. Rhee, "Dynamic Traffic Assignment with Departure Flow Estimation based on Preferred Arrival Time," KSCE Journal of Civil Engineering, vol. 16, no. 4, pp. 633-643, 2012. http://dx.doi.org/10.1007/s12205-012-1210-8

[19] Xiaoning Zhang and H. M. Zhang, "Simultaneous Departure Time/Route Choices in Queuing Networks and a Novel Paradox," Networks and Spatial Economics, vol. 10, no. 1, pp. 93-112, 2010. http://dx.doi.org/10.1007/s11067-007-9026-7

[20] Yu (Marco) Nie and H. M. Zhang, "Solving the Dynamic User Optimal Assignment Problem Considering Queue Spillback," Networks and Spatial Economics, vol. 10, no. 1, pp. 49-71, 2010. http://dx.doi.org/10.1007/s11067-007-9022-y

[21] W. Y. Szeto and S. C. Wong, "Dynamic traffic assignment: model classifications and recent advances in travel choice principles," Central European Journal of Engineering, vol. 2, no. 1, pp. 1-18, 2012. http://dx.doi.org/10.2478/s13531-011-0057-y

[22] K. L. Cooke and E. Halsey, "The shortest route through a network with time-dependent internodal transit times," Journal of mathematical analysis and applications, vol. 14, no.3, pp. 493-498, 1966. http://dx.doi.org/10.1016/0022-247X(66)90009-6

[23] T. White, Hadoop: The Definitive Guide, 3rd ed. O'Reilly Media, 2009.

[24] Transportation Network Test Problems [Online]. Available: http://www.bgu.ac.il/ bargera/tntp/ 